\title{
General Non-Extremal Rotating Black Holes in Minimal Five-Dimensional Gauged Supergravity
}

\author{
Z.-W. Chong ${ }^{1}$, M. Cvetič ${ }^{2}$, H. Lü̈${ }^{1}$, C.N. Pope ${ }^{1}$ \\ ${ }^{1}$ George P. \& Cynthia W. Mitchell Institute for Fundamental Physics, \\ Texas A\&M University, College Station, TX 7r843, USA \\ ${ }^{2}$ Department of Physics and Astronomy, University of Pennsylvania, Philadelphia, PA 19104, USA
}

(Dated: June 2, 2005)

\begin{abstract}
We construct the general solution for non-extremal charged rotating black holes in five-dimensional minimal gauged supergravity. They are characterised by four non-trivial parameters, namely the mass, the charge, and the two independent rotation parameters. The metrics in general describe regular rotating black holes, providing the parameters lie in appropriate ranges so that naked singularities and closed timelike curves (CTC's) are avoided. We calculate the conserved energy, angular momenta and charge for the solutions, and show how supersymmetric solutions arise in a BPS limit. These have naked CTC's in general, but for special choices of the parameters we obtain new regular supersymmetric black holes or smooth topological solitons.

PACS numbers: 11.25.-w, 04.50.+h
\end{abstract}

The discovery of the remarkable AdS/CFT correspondence showed that bulk properties of solutions in the five-dimensional gauged supergravities that result from compactification of the type IIB string are related to properties of strongly-coupled conformal field theories on the four-dimensional boundary of five-dimensional antide Sitter spacetime [1, 2, 3]. It therefore becomes of great importance to study the solutions of the five-dimensional gauged supergravity theories. One of the most important classes of such solutions are those that describe black holes in five dimensions. In particular, it has been argued that the boundary conformal field theory dual to rotating five-dimensional black holes should describe a system in a four-dimensional rotating Einstein universe [4].

The rotating five-dimensional solutions found in 4] were neutral Kerr-(anti)-de Sitter black holes. In order to be able to make contact with supersymmetric BPS configurations, for which the AdS/CFT correspondence is more solidly founded, it is of considerable interest to generalise the neutral solutions to include electric charge too. In the analogous problem in ungauged supergravity, it is straightforward to generate charged solutions from neutral ones, by using the global symmetries of the ungauged supergravities as solution-generating transformations. By this means, the general charged rotating black holes of five-dimensional ungauged supergravity were obtained in [5], starting from the neutral rotating Ricci-flat black holes found in [6]. For the solutions in gauged supergravity there are no surviving global symmetries that can be used to provide solution-generating transformations, and one has little option but to resort to bruteforce calculations, starting from an appropriate ansatz, to construct the charged rotating solutions. One way to simplify the problem is to specialise to the case where the two independent rotation parameters of the generic fivedimensional rotating black hole are set equal, since this reduces the problem from cohomogeneity-2, with partial differential equations, to cohomogeneity-1, with ordinary differential equations. Supersymmetric rotating black holes with two equal angular momenta were obtained in [7], and it was shown in [7] that the rotation is necessary for the solution to be free of naked singularities and CTC's. The non-extremal charged rotating solutions of gauged five-dimensional supergravity with equal rotation parameters were constructed in [8, 9]. Recently, some special cases involving unequal rotation parameters were also constructed, in 10]. However, these latter arose as solutions of $\mathcal{N}=2$ gauged supergravity coupled to two vector multiplets, with a specific relation between the three electric charges, and did not, in general, admit a specialisation to solutions of pure minimal $\mathcal{N}=2$ gauged supergravity. The purpose of this letter is to present the general solution for charged rotating non-extremal black holes in minimal five-dimensional gauged supergravity, with independent rotation parameters in the two orthogonal 2-planes.

We have found the general solution for charged rotating black holes in five-dimensional minimal gauged supergravity, with unequal angular momenta, by a process involving a considerable amount of trial and error, followed by an explicit verification that the equations of motion are satisfied. In doing this, we have been guided by the previously-obtained special case found in [8], where the two angular momenta were set equal, and the general charged rotating solutions in ungauged minimal supergravity, which are contained within the results in [5]. In this letter we begin by presenting our new solutions, and then we calculate the conserved angular momenta and electric charge. By integrating the first law of thermodynamics, we also obtain the conserved mass, or energy, of the solutions. By considering the conditions under which the anticommutator of supercharges in the AdS superalgebra has zero eigenvalues, we then show how a BPS limit 
of our general non-extremal solutions gives rise to new supersymmetric configurations. These include new supersymmetric rotating black holes, with two independentlyspecifiable angular momenta, and new topological solitons that are non-singular on complete manifolds.

In terms of Boyer-Lindquist type coordinates $x^{\mu}=$ $(t, r, \theta, \phi, \psi)$ that are asymptotically static (i.e. the coordinate frame is non-rotating at infinity), we find that the metric and gauge potential for our new rotating solutions can be expressed as

$$
\begin{aligned}
d s^{2}= & -\frac{\Delta_{\theta}\left[\left(1+g^{2} r^{2}\right) \rho^{2} d t+2 q \nu\right] d t}{\Xi_{a} \Xi_{b} \rho^{2}}+\frac{2 q \nu \omega}{\rho^{2}} \\
& +\frac{f}{\rho^{4}}\left(\frac{\Delta_{\theta} d t}{\Xi_{a} \Xi_{b}}-\omega\right)^{2}+\frac{\rho^{2} d r^{2}}{\Delta_{r}}+\frac{\rho^{2} d \theta^{2}}{\Delta_{\theta}} \\
& +\frac{r^{2}+a^{2}}{\Xi_{a}} \sin ^{2} \theta d \phi^{2}+\frac{r^{2}+b^{2}}{\Xi_{b}} \cos ^{2} \theta d \psi^{2} \\
A= & \frac{\sqrt{3} q}{\rho^{2}}\left(\frac{\Delta_{\theta} d t}{\Xi_{a} \Xi_{b}}-\omega\right),
\end{aligned}
$$

where

$$
\begin{aligned}
\nu & =b \sin ^{2} \theta d \phi+a \cos ^{2} \theta d \psi, \\
\omega & =a \sin ^{2} \theta \frac{d \phi}{\Xi_{a}}+b \cos ^{2} \theta \frac{d \psi}{\Xi_{b}}, \\
\Delta_{\theta} & =1-a^{2} g^{2} \cos ^{2} \theta-b^{2} g^{2} \sin ^{2} \theta, \\
\Delta_{r} & =\frac{\left(r^{2}+a^{2}\right)\left(r^{2}+b^{2}\right)\left(1+g^{2} r^{2}\right)+q^{2}+2 a b q}{r^{2}}-2 m, \\
\rho^{2} & =r^{2}+a^{2} \cos ^{2} \theta+b^{2} \sin ^{2} \theta, \\
\Xi_{a} & =1-a^{2} g^{2}, \quad \Xi_{b}=1-b^{2} g^{2}, \\
f & =2 m \rho^{2}-q^{2}+2 a b q g^{2} \rho^{2} .
\end{aligned}
$$

A straightforward calculation shows that these configurations solve the equations of motion of minimal gauged five-dimensional supergravity, which follow from the Lagrangian

$$
\mathcal{L}=\left(R+12 g^{2}\right) * \mathbb{1}-\frac{1}{2} * F \wedge F+\frac{1}{3 \sqrt{3}} F \wedge F \wedge A,
$$

where $F=d A$, and $g$ is assumed to be positive, without loss of generality.

For some purposes, it is useful to note that the nonvanishing metric components are given by

$$
\begin{aligned}
& g_{00}=-\frac{\Delta_{\theta}\left(1+g^{2} r^{2}\right)}{\Xi_{a} \Xi_{b}}+\frac{\Delta_{\theta}^{2}\left(2 m \rho^{2}-q^{2}+2 a b q g^{2} \rho^{2}\right)}{\rho^{4} \Xi_{a}^{2} \Xi_{b}^{2}}, \\
& g_{03}=-\frac{\Delta_{\theta}\left[a\left(2 m \rho^{2}-q^{2}\right)+b q \rho^{2}\left(1+a^{2} g^{2}\right)\right] \sin ^{2} \theta}{\rho^{4} \Xi_{a}^{2} \Xi_{b}}, \\
& g_{04}=-\frac{\Delta_{\theta}\left[b\left(2 m \rho^{2}-q^{2}\right)+a q \rho^{2}\left(1+b^{2} g^{2}\right)\right] \cos ^{2} \theta}{\rho^{4} \Xi_{b}^{2} \Xi_{a}}, \\
& g_{33}=\frac{\left(r^{2}+a^{2}\right) \sin ^{2} \theta}{\Xi_{a}}+\frac{a\left[a\left(2 m \rho^{2}-q^{2}\right)+2 b q \rho^{2}\right] \sin ^{4} \theta}{\rho^{4} \Xi_{a}^{2}}, \\
& g_{44}=\frac{\left(r^{2}+b^{2}\right) \cos ^{2} \theta}{\Xi_{b}}+\frac{b\left[b\left(2 m \rho^{2}-q^{2}\right)+2 a q \rho^{2}\right] \cos ^{4} \theta}{\rho^{4} \Xi_{b}^{2}},
\end{aligned}
$$

$$
\begin{aligned}
& g_{34}=\frac{\left[a b\left(2 m \rho^{2}-q^{2}\right)+\left(a^{2}+b^{2}\right) q \rho^{2}\right] \sin ^{2} \theta \cos ^{2} \theta}{\rho^{4} \Xi_{a} \Xi_{b}} \\
& g_{11}=\frac{\rho^{2}}{\Delta_{r}}, \quad g_{22}=\frac{\rho^{2}}{\Delta_{\theta}} .
\end{aligned}
$$

The Killing vector

$$
\ell=\frac{\partial}{\partial t}+\Omega_{a} \frac{\partial}{\partial \phi}+\Omega_{b} \frac{\partial}{\partial \psi}
$$

becomes null on the outer Killing horizon at $r=r_{+}$, the largest positive root of $\Delta_{r}=0$, where the angular velocities on the horizon are given by

$$
\begin{aligned}
& \Omega_{a}=\frac{a\left(r_{+}^{2}+b^{2}\right)\left(1+g^{2} r_{+}^{2}\right)+b q}{\left(r_{+}^{2}+a^{2}\right)\left(r_{+}^{2}+b^{2}\right)+a b q}, \\
& \Omega_{b}=\frac{b\left(r_{+}^{2}+a^{2}\right)\left(1+g^{2} r_{+}^{2}\right)+a q}{\left(r_{+}^{2}+a^{2}\right)\left(r_{+}^{2}+b^{2}\right)+a b q} .
\end{aligned}
$$

One can then easily evaluate the surface gravity

$$
\kappa=\frac{r_{+}^{4}\left[\left(1+g^{2}\left(2 r_{+}^{2}+a^{2}+b^{2}\right)\right]-(a b+q)^{2}\right.}{r_{+}\left[\left(r_{+}^{2}+a^{2}\right)\left(r_{+}^{2}+b^{2}\right)+a b q\right]},
$$

and hence the Hawking temperature $T=\kappa /(2 \pi)$. The entropy is given by

$$
S=\frac{\pi^{2}\left[\left(r_{+}^{2}+a^{2}\right)\left(r_{+}^{2}+b^{2}\right)+a b q\right]}{2 \Xi_{a} \Xi_{b} r_{+}}
$$

The angular momenta can be evaluated from the Komar integrals $J=1 /(16 \pi) \int_{S^{3}} * d K$, where $K=\partial / \partial \phi$ or $K=\partial / \partial \psi$, yielding

$$
\begin{aligned}
& J_{a}=\frac{\pi\left[2 a m+q b\left(1+a^{2} g^{2}\right)\right]}{4 \Xi_{a}^{2} \Xi_{b}} \\
& J_{b}=\frac{\pi\left[2 b m+q a\left(1+b^{2} g^{2}\right)\right]}{4 \Xi_{b}^{2} \Xi_{a}} .
\end{aligned}
$$

The electric charge follows from the Gaussian integral $Q=1 /(16 \pi) \int_{S^{3}}(* F-F \wedge A / \sqrt{3})$, yielding

$$
Q=\frac{\sqrt{3} \pi q}{4 \Xi_{a} \Xi_{b}}
$$

Using the technique introduced in [11], the easiest way to calculate the conserved mass, or energy, is to integrate the first law of thermodynamics $d E=T d S+\Omega_{a} d J_{a}+$ $\Omega_{b} d J_{b}+\Phi d Q$, where $\Phi=\ell^{\mu} A_{\mu}$ is the electrostatic potential on the horizon. Doing this, we find

$$
E=\frac{m \pi\left(2 \Xi_{a}+2 \Xi_{b}-\Xi_{a} \Xi_{b}\right)+2 \pi q a b g^{2}\left(\Xi_{a}+\Xi_{b}\right)}{4 \Xi_{a}^{2} \Xi_{b}^{2}}
$$

The BPS limit can be found by looking at the eigenvalues of the Bogomol'nyi matrix coming from the anticommutators of the supercharges, as discussed in [12]. Thus we have BPS solutions if

$$
E-g J_{a}-g J_{b}-\sqrt{3} Q=0 .
$$


From the expressions derived above for $\left(E, J_{a}, J_{b}, Q\right)$, we find that the BPS limit is achieved if

$$
q=\frac{m}{1+(a+b) g}
$$

The supersymmetry of the solutions in this limit can be confirmed by calculating the norm of the Killing vector

$$
K_{+} \equiv \frac{\partial}{\partial t}+g \frac{\partial}{\partial \phi}+g \frac{\partial}{\partial \psi},
$$

which, as discussed in [12], arises as the square of the Killing spinor $\eta$, in the sense that $K_{+}^{\mu}=\bar{\eta} \gamma^{\mu} \eta$. We find that its norm is given by

$$
K_{+}^{2}=-\frac{\left[h-m\left(1+a g \cos ^{2} \theta+b g \sin ^{2} \theta\right)\right]^{2}}{h^{2}},
$$

where

$$
h=(1+a g)(1+b g)[1+(a+b) g] \rho^{2} .
$$

Thus indeed the norm of $K_{+}$is, as it should be since it has a spinorial square root, manifestly negative definite. The fraction of supersymmetry preserved is in general $\frac{1}{4}$, except when $a=-b$, in which case, the preserved supersymmetry is doubled to become $\frac{1}{2}$. The latter solution was previously obtained in [13].

We now discuss the global structure of the rotating $\mathrm{AdS}_{5}$ black hole. To do this, we first note that the metric can be expressed as

$$
\begin{aligned}
d s^{2}= & -\frac{\Delta_{r} \Delta_{\theta} r^{2} \sin ^{2} 2 \theta}{4 \Xi_{a}^{2} \Xi_{b}^{2} B_{\phi} B_{\psi}} d t^{2}+\rho^{2}\left(\frac{d r^{2}}{\Delta_{r}}+\frac{d \theta^{2}}{\Delta_{\theta}}\right) \\
& +B_{\psi}\left(d \psi+v_{1} d \phi+v_{2} d t\right)^{2}+B_{\phi}\left(d \phi+v_{3} d t\right)^{2}
\end{aligned}
$$

where the functions $B_{\phi}, B_{\psi}, v_{1}, v_{2}$ and $v_{3}$ can be straightforwardly found by comparing (18) with the metric in (11). The absence of naked closed timelike curves (CTC's) requires that $B_{\phi}$ and $B_{\psi}$ be non-negative outside the horizon. We shall focus on the discussion of supersymmetric solutions, satisfying the condition (14). It can be seen from (16) that the identity

$$
\begin{aligned}
& -\frac{\Delta_{r} \Delta_{\theta} r^{2} \sin ^{2} 2 \theta}{4 \Xi_{a}^{2} \Xi_{b}^{2} B_{\phi} B_{\psi}}+B_{\psi}\left(v_{2}+g+g v_{1}\right)^{2}+B_{\phi}\left(v_{3}+g\right)^{2} \\
& =-\frac{\left[h-m\left(1+a g \cos ^{2} \theta+b g \sin ^{2} \theta\right)\right]^{2}}{h^{2}}
\end{aligned}
$$

holds. It follows that in general, at the Killing horizon where $\Delta_{r}=0$, we have $B_{\phi} \cdot B_{\psi}<0$, implying the existence of naked CTC's. There are two special cases where naked CTC's can be avoided, leading to either supersymmetric black holes or topological solitons:

Supersymmetric black holes: The first way to avoid naked CTC's is if the right-hand side of (19) vanishes on the
Killing horizon. This occurs when the parameters in the supersymmetric solutions satisfy the further restriction

$$
g m=(a+b)(1+a g)(1+b g)(1+a g+b g) .
$$

Remarkably, when this extra condition is satisfied, the function $\Delta_{r}$ has a double root; $\Delta_{r}$ is now given by

$$
\Delta_{r}=r^{-2}\left(r^{2}-r_{0}^{2}\right)^{2}\left[g^{2} r^{2}+(1+a g+b g)^{2}\right],
$$

where $r_{0}^{2}=g^{-1}(a+b+a b g)$. At the Killing horizon $r=r_{0}$, we find that the determinant of the metric in the $(\theta, \phi, \psi)$ directions is given by

$$
\operatorname{det} g_{(\theta, \phi, \psi)}=\frac{(a+b)^{2}(a+b+a b g) \sin ^{2} 2 \theta}{4 g^{3}(1-a g)^{2}(1-b g)^{2}}
$$

This implies that naked CTC's are avoided if the remaining free parameters $a$ and $b$ satisfy the inequality

$$
a+b+a b g>0
$$

The Killing horizon $r=r_{0}$ is then the event horizon of a well-defined supersymmetric black hole that is regular on and outside the event horizon. The occurrence of the double-root of $\Delta_{r}$ at $r=r_{0}$ implies that the black hole has zero temperature. The various conserved and thermodynamic quantities for these new supersymmetric black holes are given by

$$
\begin{aligned}
E= & \frac{\pi(a+b)}{4 g(1-a g)^{2}(1-b g)^{2}}((1-a g)(1-b g) \\
& \quad+(1+a g)(1+b g)(2-a g-b g)), \\
S= & \frac{\pi^{2}(a+b) \sqrt{a+b+a b g}}{2 g^{3 / 2}(1-a g)(1-b g)} \\
J_{a}= & \frac{\pi(a+b)(2 a+b+a b g)}{4 g(1-a g)^{2}(1-b g)}, \\
J_{b}= & \frac{\pi(a+b)(a+2 b+a b g)}{4 g(1-a g)(1-b g)^{2}}, \\
Q= & -\frac{\pi \sqrt{3}(a+b)}{4 g(1-a g)(1-b g)} .
\end{aligned}
$$

Note that supersymmetric black holes cannot arise when $a=-b$. For $a=b$, our new supersymmetric black hole solutions, which for general $a$ and $b$ have cohomogeneity 2 , become cohomogeneity 1 ; these special cases were previously obtained in [7].

Topological solitons: The second way to avoid naked CTC's is if $B_{\phi}=0$ at $r=r_{0}$. This can happen when the free parameters in the general supersymmetric solutions obey the further restriction

$$
\begin{aligned}
m=-(1 & +a g)(1+b g)(1+a g+b g) \\
& \times(2 a+b+a b g)(a+2 b+a b g) .
\end{aligned}
$$


Now $r_{0}$, the outer root of $\Delta_{r}$, is given by

$$
r_{0}^{2}=-(a+b+a b g)^{2} .
$$

Defining a new radial coordinate $R=r^{2}-r_{0}^{2}$, we find that the metric describes a smooth topological soliton, with $R$ running from 0 to $\infty$. The requirement of the absence of a conical singularity when $B_{\phi}$ vanishes at $R=0$ implies the quantisation condition

$$
\frac{(a+b+a b g)\left(3+5 a g+5 b g+3 a b g^{2}\right)}{(1-a g)(a+2 b+a b g)}=1 .
$$

In the cohomogeneity- 1 special cases $a=b$ or $a=-b$, these toplogical solitons are encompassed within the soliton solutions obtained in [12].

Aside from the above two possibilities, the supersymmetric solutions in general have naked CTC's. As in the examples discussed in [10,12], a conical singularity at the Killing horizon can be avoided by periodically identifying the asymptotic time coordinate $t$ with an appropriate period. However, if the Killing horizon is associated with a double root of $\Delta_{r}$, then such an identification is unnecessary, analogous to the ungauged rotating solution obtained in [14]. The geodesic analysis of analogous time machines can be found in 15,16 .

In the general case where the charged rotating metrics that we have found are non-extremal, they describe regular black holes provided the parameters lie in appropriate ranges that are easily determinable using the same techniques we have used above for analysing the BPS limits.

As discussed in [4], rotating black hole solutions in fivedimensional gauged supergravity provide backgrounds whose AdS/CFT duals describe four-dimensional field theories in the rotating Einstein universe on the boundary of anti-de Sitter spacetime. With the general solutions in minimal gauged supergravity that we have now found, this aspect of the AdS/CFT correspondence can be studied in a framework that also allows one to take a BPS or near-BPS limit, where the mapping from the bulk to the boundary is better controlled. In particular, it is of great interest to provide the microscopic interpretation from the boundary CFT for the entropy (24) of the supersymmetric black holes with two general rotations. We plan to report further on these considerations in forthcoming work.

\section{Acknowledgements:}

We thank Gary Gibbons for useful discussions. C.N.P. thanks the Relativity and Cosmology group in DAMTP,
Cambridge, for hospitality during the course of this work. Research supported in part by DOE grants DEFG02-95ER40893 and DE-FG03-95ER40917, NSF grant INTO3-24081, and (M.C.) the University of Pennsylvania Research Foundation Award and the Fay R. and Eugene L.Langberg Chair.

[1] J.M. Maldacena, The large $N$ limit of superconformal field theories and supergravity, Adv. Theor. Math. Phys. 2, 231 (1998), hep-th/9711200

[2] S.S. Gubser, I.R. Klebanov and A.M. Polyakov, Gauge theory correlators from non-critical string theory, Phys. Lett. B428, 105 (1998), hep-th/9802109

[3] E. Witten, Anti-de Sitter space and holography, Adv. Theor. Math. Phys. 2, 253 (1998), hep-th/9802150

[4] S.W. Hawking, C.J. Hunter and M.M. Taylor-Robinson, Rotation and the AdS/CFT correspondence, Phys. Rev. D59, 064005 (1999), hep-th/9811056

[5] M. Cvetič and D. Youm, General rotating five dimensional black holes of toroidally compactified heterotic string, Nucl. Phys. B476, 118 (1996), hep-th/9603100.

[6] R.C. Myers and M.J. Perry, Black holes in higher dimensional space-times, Annals Phys. 172, 304 (1986).

[7] J.B. Gutowski and H.S. Reall, Supersymmetric $A d S_{5}$ black holes, JHEP 0402, 006 (2004), hep-th/0401042

[8] M. Cvetič, H. Lü and C.N. Pope, Charged Kerr-de Sitter black holes in five dimensions, Phys. Lett. B598, 273 (2004), hep-th/0406196

[9] M. Cvetič, H. Lü and C.N. Pope, Charged rotating black holes in five dimensional $U(1)^{3}$ gauged $N=2$ supergravity, Phys. Rev. D70, 081502 (2004), hep-th/0407058

[10] Z.W. Chong, M. Cvetič, H. Lü and C.N. Pope, Fivedimensional gauged supergravity black holes with independent rotation parameters,

[11] G.W. Gibbons, M.J. Perry and C.N. Pope, The first law of thermodynamics for Kerr-anti-de Sitter black holes, hep-th/0408217 to appear in Class. Quantum Grav.

[12] M. Cvetič, G.W. Gibbons, H. Lü and C.N. Pope, Rotating black holes in gauged supergravities: Thermodynamics, supersymmetric limits, topological solitons and time machines, hep-th/0504080

[13] D. Klemm and W.A. Sabra, Charged rotating black holes in 5d Einstein-Maxwell-(A)dS gravity, Phys. Lett. B 503, 147 (2001), hep-th/0010200

[14] J.C. Breckenridge, R.C. Myers, A.W. Peet and C. Vafa, D-branes and spinning black holes, Phys. Lett. B391, 93 (1997), hep-th/9602065

[15] G.W. Gibbons and C.A.R. Herdeiro, Supersymmetric rotating black holes and causality violation, Class. Quant. Grav. 16, 3619 (1999), hep-th/9906098

[16] M.M. Caldarelli, D. Klemm and W.A. Sabra, Causality violation and naked time machines in $A d S_{5}$, JHEP 0105, 014 (2001), hep-th/0103133 